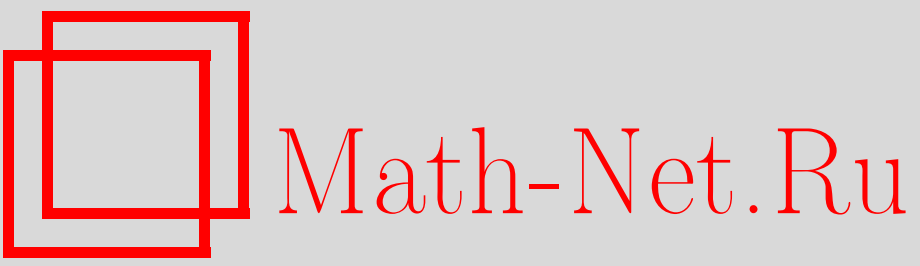

Д. Н. Иванов, Адамаровы разложения полупростых ассоциативных алгебр, УМH, 2003, том 58, выпуск 4, 147-148

DOI: https://doi.org/10.4213/rm648

Использование Общероссийского математического портала Math-Net.Ru подразумевает, что вы прочитали и согласны с пользовательским соглашением

http://www.mathnet.ru/rus/agreement

Параметры загрузки:

IP: 52.87 .193 .239

26 апреля 2023 г., 16:17:56

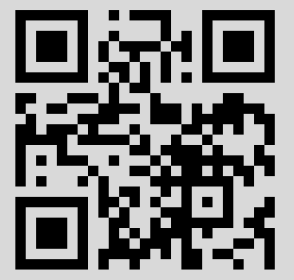




\section{АДАМАРОВЫ РАЗЛОЖЕНИЯ ПОЛУПРОСТЫХ АССОЦИАТИВНЫХ АЛГЕБР}

\section{Д. Н. ИвАнОВ}

Теорема 2 [1] утверждает, что прямая сумма двух адамаровых алгебр одинаковой размерности является адамаровой алгеброй. В данной работе этот результат обобщается на случай прямой суммы $n$ адамаровых алгебр, где $n$ - порядок некоторой матрицы Адамара. Кроме этого, дается отрицательный ответ на вопрос из [1] - является ли прямая сумма двух матричных алгебр нечетной размерности адамаровой алгеброй. Это подтверждает гипотезу о делимости размерности адамаровой алгебры на 4.

Напомним основные определения. Пусть $\mathbf{A}$ - ассоциативная конечномерная над полем комплексных чисел $\mathbb{C}$ алгебра, через $\operatorname{Tr}_{A}$ обозначим след правого регулярного представления $\mathbf{A}$. Тогда $\operatorname{Tr}_{A} x y$ является симметричной инвариантной формой на $\mathbf{A}$, которую мы будем называть фор мой следа. Известно, что алгебра $\mathbf{A}$ полупроста тогда и только тогда, когда форма следа невьрождена на А. Далее будут рассматриваться полупростые алгебры и термин "алгебра", если не оговорено противное, будет обозначать ассоциативную конечномерную над $\mathbb{C}$ полупростую алгебру. Таким образом, по теореме Веддербарна алгебра $\mathbf{A}$ изоморфна конечной прямой сумме матричных алгебр $\mathrm{M}_{n}(\mathbb{C})=\mathrm{M}_{n}$.

ОПРЕДЕЛЕнИЕ 1. Семейство неединичных собственных подалгебр $\mathscr{D}=\left\{\mathbf{B}_{i}, i=1, \ldots, r\right\}$ образует ортогональное разложение (ОР) алгебры $\mathbf{A}$, если

(1) все подалгебры $\mathbf{B}_{i}$ полупросты и содержат единичный элемент $\mathbf{1}_{A}$ алгебры $\mathbf{A}$;

(2) (условие ортогональности) алгебра $\mathbf{A}$ является прямой суммой попарно ортогональных подпространств

$$
\mathbf{A}=\left\langle\mathbf{1}_{A}\right\rangle \oplus \mathbf{B}_{1}^{\circ} \oplus \cdots \oplus \mathbf{B}_{r}^{\circ},
$$

где $\mathbf{B}_{i}^{\circ}=\left\{x \in \mathbf{B}_{i} \mid \operatorname{Tr}_{A} x=0\right\}$.

Если все подалгебры ОР $\mathscr{D}$ коммутативны, то $\mathscr{D}$ назьвается коммутативныл. Если все подалгебры в $\mathscr{D}$ изоморфны алгебре $\mathbf{B}$, то $\mathscr{D}$ назьвается однородныц $O P$ muпа $\mathbf{B}$ (или просто OP muna $\mathbf{B})$.

Через $k \mathrm{M}_{n}$ будем обозначать прямую сумму $k$ экземпляров алгебры матриц $\mathrm{M}_{n}$.

Однородное $\mathrm{OP}$ типа $2 \mathrm{M}_{1}$ назьвается адамаровым разложением. Алгебра, допускающая адамарово разложение, назьвается адамаровой.

Нам понадобится следующий результат из [1].

Лемма 1. Пусть подалгебры $\left\{\mathbf{B}_{1}, \ldots, \mathbf{B}_{r}\right\}$ образуют адамарово разложсение алгебры А. Пусть $\operatorname{Pr}\left(\mathbf{B}_{i}\right)=\left\{e_{i 1}, e_{i 2}\right\}-$ примитивные идемпотенты подалгебры $\mathbf{B}_{i}, i=\overline{1, r}$. Тогда

(1) число подалгебр в адамаровом разложении на единичу меньше размерности алгебры, m.е. $r=\operatorname{dim} \mathbf{A}-1$;

(2) $\operatorname{Tr}_{A} e_{i 1}=\operatorname{Tr}_{A} e_{i 2}=\frac{1}{2} \operatorname{dim} \mathbf{A}$ для любого $i=\overline{1, r}$, в частности, размерность адамаровой алгебры четна;

(3) в каждой подалгебре адамарова разложения содержится ровно две инволючии с нулевым следом регулярного представления, причем эти инволюции, взятые по одной из всех подалгебр разложения, образуют базис подпространства $\mathbf{A}^{\circ}=$ $\left\{x \in \mathbf{A} \mid \operatorname{Tr}_{A} x=0\right\}$.

ТеОрема 1. Прямая сумма $n$ адамаровых алгебр одинаковой размерности является адамаровой алгеброй, если существует матрица Адамара порядка $n$.

Работа выполнена при поддержке Российского фонда фундаментальных исследований (грант № 02-01-00219). 
ДоказАтельство. Пусть $\mathbf{A}=\mathbf{A}_{1} \oplus \cdots \oplus \mathbf{A}_{n}$ - прямая сумма адамаровых алгебр $\mathbf{A}_{i}$ одинаковой размерности $\operatorname{dim} \mathbf{A}_{i}=m$. Тогда для $x=\left(x_{1}, \ldots, x_{n}\right) \in \mathbf{A}: \operatorname{Tr}_{A} x=\operatorname{Tr}_{A_{1}} x_{1}+\cdots+$ $\operatorname{Tr}_{A_{n}} x_{n}$.

Пусть инволюции $a_{j}(i)$ порождают подалгебры адамарова разложения в алгебре $\mathbf{A}_{i}, j=$ $1, \ldots, m-1, i=1, \ldots, n$. В силу леммы $1, \operatorname{Tr}_{A_{i}} a_{j}(i)=0$. Пусть $H=\left(h_{i j}\right)$ - нормализованная матрица Адамара порядка $n$, т.е. $h_{i j}= \pm 1$ и $H \cdot H^{t}=E_{n}\left(E_{n}\right.$ - единичная матрица), причем первая строка (и столбец) матрицы $H$ состоят из одних единиц. Рассмотрим следующую систему инволюций:

$$
\begin{gathered}
b_{j s}=\left(h_{s 1} a_{j}(1), \ldots, h_{s n} a_{j}(n)\right), \quad j=1, \ldots, m-1, \quad s=1, \ldots, n ; \\
c_{i}=\left(h_{i 1} \mathbf{1}_{A_{1}}, \ldots, h_{i n} \mathbf{1}_{A_{n}}\right), \quad i=2, \ldots, n .
\end{gathered}
$$

Легко видеть, что эти инволюции ортогоналњны единичному элементу $\mathbf{1}_{A}$ и попарно ортогоналшны (относителшно формы следа). Так как их число равно $(m-1) n+(n-1)=m n-1=$ $\operatorname{dim} \mathbf{A}-1$, то подалгебры, порожденные указанньми инволюциями, образуют адамарово разложение алгебры $\mathbf{A}$. Теорема доказана.

Теорема 2. Если алгебра $\mathrm{M}_{n_{1}} \oplus \mathrm{M}_{n_{2}}$ адамарова, то $n_{1}$ и $n_{2}$ четны.

ДокАЗАТЕЛЬСтво. Пусть $\mathbf{A}=\mathrm{M}_{n_{1}} \oplus \mathrm{M}_{n_{2}}$ - адамарова алгебра. Положим $n=\operatorname{dim} \mathbf{A}=$ $n_{1}^{2}+n_{2}^{2}$ и $\operatorname{Tr}_{A}=\operatorname{Tr}$. В силу леммы $1, n$ четно. Для $X=\left(X_{1}, X_{2}\right) \in \mathbf{A}$ имеем

$$
\operatorname{Tr} X=n_{1} \operatorname{tr} X_{1}+n_{2} \operatorname{tr} X_{2}
$$

где tr обозначает след матрицы.

Случай $n_{1}=n_{2}=1$ тривиален, так как очевидно, что алгебра $\mathrm{M}_{1} \oplus \mathrm{M}_{1}$ неадамарова. Поэтому далее без ограничения общности предполагаем $n_{1}>1$.

Инволюции $A_{i}=\left(A_{i 1}, A_{i 2}\right), i=1, \ldots, n-1$, выбранные по одной из каждой подалгебры адамарова разложения, вместе с единичньм элементом $\mathbf{1}_{A}$ в силу леммы 1 образуют ортогональньй базис алгебры $\mathbf{A}$. Следовательно, для любого $X=\left(X_{1}, X_{2}\right) \in \mathbf{A}$

$$
n \operatorname{Tr} X^{2}=\sum_{i=1}^{n-1}\left(\operatorname{Tr} X A_{i}\right)^{2}+(\operatorname{Tr} X)^{2} .
$$

Подставляя в (1) $X=\left(E_{n_{1}}, 0\right)$, где $E_{n_{1}}$ - единичная матрица, получим

$$
n n_{1}^{2}=\sum_{i=1}^{n-1}\left(\operatorname{Tr} A_{i 1}\right)^{2}+n_{1}^{4} .
$$

Отсюда, после сокращения на $n_{1}^{2}$ и учитьвая, что $n=n_{1}^{2}+n_{2}^{2}$, выводим

$$
n_{2}^{2}=\sum_{i=1}^{n-1}\left(\operatorname{tr} A_{i 1}\right)^{2} .
$$

Так как след инволютивной матрицы есть целое число, то в силу неравенства $n-1=n_{1}^{2}+$ $n_{2}^{2}-1>n_{2}^{2}$ получаем, что для некоторого $i: \operatorname{tr} A_{i 1}=0$. Но след инволютивной матрицы $A_{i 1}$ может быть равен нулю, только если ее порядок $n_{1}$ четен. Значит, $n_{2}^{2}=n-n_{1}^{2}$ также четно. Теорема доказана.

ЗАмечаниЕ. Автор предполагает, что если алгебра $\mathbf{A}=\mathrm{M}_{n_{1}} \oplus \mathrm{M}_{n_{2}}$ адамарова, то $n_{1}=n_{2}$ и любое адамарово разложение алгебры $\mathbf{A}$ содержит ее центральную двумерную подалгебру.

Автор благодарен проф. М.В.Зайцеву за поддержку и внимание к работе.

\section{СПИСОК ЛИТЕРАТУРЫ}

[1] Д. Н. Иванов // Матем. сб. 1998. Т. 189. №12. С. 83-102. 